\title{
Punción percutánea transtorácica con aguja gruesa: complicaciones y factores de riesgo inherentes al paciente
}

\section{Imaging-guided Chest Biopsies with Thick Needle: Complications and Risk Factors Inherent to the Patient}

\author{
Ricardo Cobeñas ${ }^{1}$ Jairo Hernandez Pinzon ${ }^{2}$ Ricardo Re $^{3}$ Karen Mena ${ }^{4}$ Mariangela Paba ${ }^{4}$ \\ Nebil Larrañaga ${ }^{1}$ Shigeru Kozima ${ }^{1}$ \\ ${ }^{1}$ Especialista en Diagnóstico por Imágenes, CEMIC, Buenos Aires, Argentina
2 Magíster en Epidemiología, CEMIC, Buenos Aires, Argentina
${ }^{3}$ Especialista en Neumología, CEMIC, Buenos Aires, Argentina
${ }^{4}$ Residente 2do año Diagnóstico por Imágenes, CEMIC, Buenos Aires,
Argentina \\ Address for correspondence Jairo Hernández Pinzón, \\ Departamento de Imágenes, Centro de Educación Medica e \\ Investigaciones Clínicas, Hospital Universitario Sede Saavedra, Galván \\ 4102, Capital Federal, Argentina \\ (e-mail: jahernandezpinzon@gmail.com).
}

Rev Argent Radiol 2018;82:154-160.

\section{Resumen \\ Palabras Clave \\ - radiología intervencionista \\ - percutánea \\ - biopsia \\ - cáncer de pulmón}

\section{Abstract}

Objetivo Determinar la incidencia de complicaciones con el uso de una aguja gruesa (semiautomática Trucut 18), para punción pulmonar y realizar un análisis exploratorio de las variables inherentes al paciente que probablemente puedan tener relación con el desarrollo de complicaciones.

Materiales y Métodos Estudio retrospectivo de una serie de casos de pacientes sometidos a punción percutánea transtorácica (PPT) con aguja gruesa y guiada por tomografía computada (TC) para el estudio de lesiones pulmonares; se realizó un análisis univariado.

Resultados Se realizaron 26 punciones, la tasa de incidencia de complicaciones fue del 38,46\% en 1 año; los pacientes presentaron: neumotórax leve $(n=7)$, neumotórax moderado $(n=3)$ y hemorragia alveolar difusa $(n=1)$. El análisis estadístico univariado mostró una diferencia estadísticamente significativa en la edad de los pacientes que presentaron complicaciones $\mathrm{v} / \mathrm{s}$ los pacientes que no presentaron complicaciones $(61,18+\mid-3,6$ versus $75,1+\mid-2,46$ años, $p=0,0107)$.

Conclusión La PPT-TC es un procedimiento con una tasa considerable de complicaciones no severas; en nuestra serie de casos, la edad fue la variable que se asoció con mayor fuerza al probable desarrollo de complicaciones.

Objective Establish the incidence of complications with the use of thick needle (Trucut 18) for pulmonary puncture and perform an exploratory analysis of the inherent variables to the patient that may be related to complication development. Materials and Methods Retrospective study of a case series of patients undergoing transthoracic percutaneous puncture (PPT) with thick needle,guided by computed received

April 7, 2018

accepted

November 20, 2018
DOI https://doi.org/

10.1055/s-0038-1676785. ISSN 1852-9992.
Copyright (c) 2019, Sociedad Argentina de Radiología. Publicado por Thieme Revinter Publicações Ltda., Rio de Janeiro, Brazil. Todos los derechos reservados.
License terms

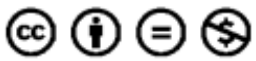




\author{
Keywords \\ - interventional \\ radiology \\ - percutaneous \\ - biopsy \\ - lung cancer
}

tomography (CT) for the study of pulmonary lesions; a univariate and multivariate analysis was performed.

Results 26 punctures were performed, the incidence rate of complications was $38.46 \%$ in 1 year: patients presented: mild pneumothorax $(n=7)$, moderate pneumothorax $(n=3)$ and diffuse alveolar hemorrhage $(n=1)$. The univariate statistical analysis showed a statistically significant difference in the age of the patients who presented complications $\mathrm{v} / \mathrm{s}$ patients who did not present complications $(61.18+/-3.6$ versus $75.1+/-2.46$ years, $p=0.0107)$.

Conclusion PPT-CT is a procedure with a considerable rate of non-severe complications; in our case series, age was the variable that was most strongly associated with the probable development of complications.

\section{Introducción}

En la era de la quimioterapia personalizada, la punción percutánea transtorácica (PPT) guiada por tomografía computada (TC), juega cada día un rol más importante en el manejo del cáncer de pulmón (CaP), ${ }^{1}$ más aún hoy en día, en que debido a los programas de screening de CaP se proyecta un incremento significativo en el número y complicaciones de PPT-TC. ${ }^{2}$

Desde la introducción de los inhibidores de la quinasa de tirosina como opción terapéutica para el adenocarcinoma de pulmón, las pruebas moleculares auxiliares han ganado importancia, debido a que la presencia o ausencia de ciertas mutaciones como el epidermal growth factor receptor (EGFR), el anaplastic large cell lymphoma kinase (ALK), o la amplificación del hepatocyte growth factor receptor (MET), ayudan no solo a predecir la respuesta, sino también a determinar el pronóstico de la enfermedad. ${ }^{3}$ Es por eso que el College of American Pathologists, la International Association for the Study of Lung Cancer y la Association for Molecular Pathology, recomiendan estudiar la presencia de ALK y EGFR en todos los pacientes con adenocarcinoma pulmonar en estadios avanzados; ${ }^{4}$ sin embargo, para realizar esos estudios moleculares, se requieren muestras de tejido adecuadas.

Los especímenes quirúrgicos proveen abundante tejido tumoral para realizar esas pruebas, pero por lo general a los $\mathrm{CaP}$ en estadios avanzados no se les realiza resección quirúrgica y en consecuencia se acude al especialista en radiología intervencionista para la obtención de muestras por vía percutánea (PPT-CT).

Es en ese punto donde radica el dilema, entre realizar ese procedimiento mediante aguja gruesa (sensibilidad, especificidad y exactitud del $89 \%, \quad 97 \%$ y $\quad 93 \%$ respectivamente ${ }^{5}$ ), que tiene una alta tasa de complicaciones (principalmente neumotórax y hemorragia pulmonar ${ }^{1}$ ) versus aguja fina (sensibilidad hasta del 99\%, especificidad y exactitud de hasta el $100 \%$ y $97 \%$ respectivamente ${ }^{6}$ ), teniendo en cuenta la limitación de que, aunque el material obtenido con esa técnica puede ser aceptable para realizar el diagnóstico, en muchas oportunidades es insuficiente para realizar el análisis molecular $^{3}$ como lo reportaron Arcila y col. ${ }^{7}$ (89\% de éxito para el test de EGFR con biopsia v/s 79\% con aguja fina).

En nuestra institución desde hace aproximadamente más de un año, por solicitud de los servicios de oncología y patología, hemos optado por realizar las PPT-TC con aguja gruesa; el objetivo de este trabajo es describir la tasa de incidencia de complicaciones que hemos tenido y los principales factores de riesgo inherentes al paciente relacionadas con las complicaciones en la población atendida desde el 2017 hasta la fecha.

\section{Materiales y Métodos}

Sobre una cohorte de pacientes sometidos a punciones pulmonares por sospecha de CaP, realizamos un estudio analítico retrospectivo con casos anidados de pacientes a quienes se les realizó el procedimiento con aguja gruesa entre enero del 2017 y febrero del 2018.

Se recolectó la información en una hoja de cálculo del programa Microsoft Excel y posteriormente se analizaron los datos mediante el programa estadístico Stata 15 (StataCorp LLC, CollegeStation Texas). Las variables continuas se reportan mediante estadísticos de tendencia central, las variables nominales y ordinales se reportan mediante porcentajes y frecuencias, la tasa de incidencia se reporta medida en un periodo de un año de observación, se dividieron los pacientes en dos grupos: con complicaciones (neumotórax y hemorragia) y sin complicaciones. Realizamos comparaciones entre variables nominales y ordinales con la prueba de chi2 o la prueba exacta de Fisher de acuerdo al número de pacientes analizados en cada celda de las tablas de contingencia y entre variables continuas mediante el $t$-test.

\section{Criterios de Inclusión y Exclusión}

Dentro de los criterios de inclusión, se tomaron en cuenta pacientes con nódulo, masa o consolidación pulmonar, pruebas de coagulación dentro de los parámetros normales realizadas no más allá de 4 días previo a la biopsia y pacientes que tolerasen decúbito supino o decúbito prono. 
Los criterios de exclusión fueron lesiones menores a $5 \mathrm{~mm}$, hipertensión pulmonar y la negativa a dar consentimiento informado para el procedimiento.

\section{Método de Imagen}

Los procedimientos fueron realizados en un tomógrafo Philips Mx 16 (Cleveland, Ohio, USA) de 16 detectores, todos los pacientes contaban con estudios de tomografía computada previos, por lo que los parámetros de adquisición de las imágenes se optimizaron, realizando una primera adquisición localizada en la zona previamente conocida de $1 \mathrm{~mm}$ cada 0,5 mm de espesor, FOV 340-450, matriz de 512, Kv 120 mA 100; una vez identificada la lesión pulmonar, tomadas las medidas y marcado el paciente, se realizaron las subsiguientes series de $3 \mathrm{~mm}$ cada $3 \mathrm{~mm}$, aproximadamente 8 cortes con el mismo FOV por serie, trabajándose con un filtro estándar para pulmón. Realizamos entonces la reconstrucción en cortes axiales, sagitales y coronales para guiar el sitio de la PPT-TC, la dirección y el punto hasta donde avanzar la aguja.

\section{Método de la Punción}

Todas las punciones fueron realizadas por un médico especialista en radiología con experiencia de 5 años en el campo del intervencionismo percutáneo guiado por imágenes (RC) con la ayuda de residentes de 4to año. En todos los procedimientos se realizó la discusión de los riesgos y beneficios antes de firmar el consentimiento informado. Se contó también, con la presencia de un residente de patología que examinó las muestras de tejido obtenidas, informando en el momento si ellas eran útiles para el diagnóstico o si se requería mayor cantidad de material. En la - Fig. 1 se describe en detalle los pasos que realizamos en cada una de las punciones.

En caso de evidencia de neumotórax moderado, definido como el colapso de la superficie pleural más de $2 \mathrm{~cm}$ desde el sitio de la punción, el mismo fue aspirado percutáneamente. Se realizó observación activa de cada paciente durante 3 horas, durante ese tiempo, se tomaron las constantes vitales cada 30 minutos y al final de ese lapso, si el paciente se encontraba asintomático era dado de alta; o en caso de presentar neumotórax se realizaba
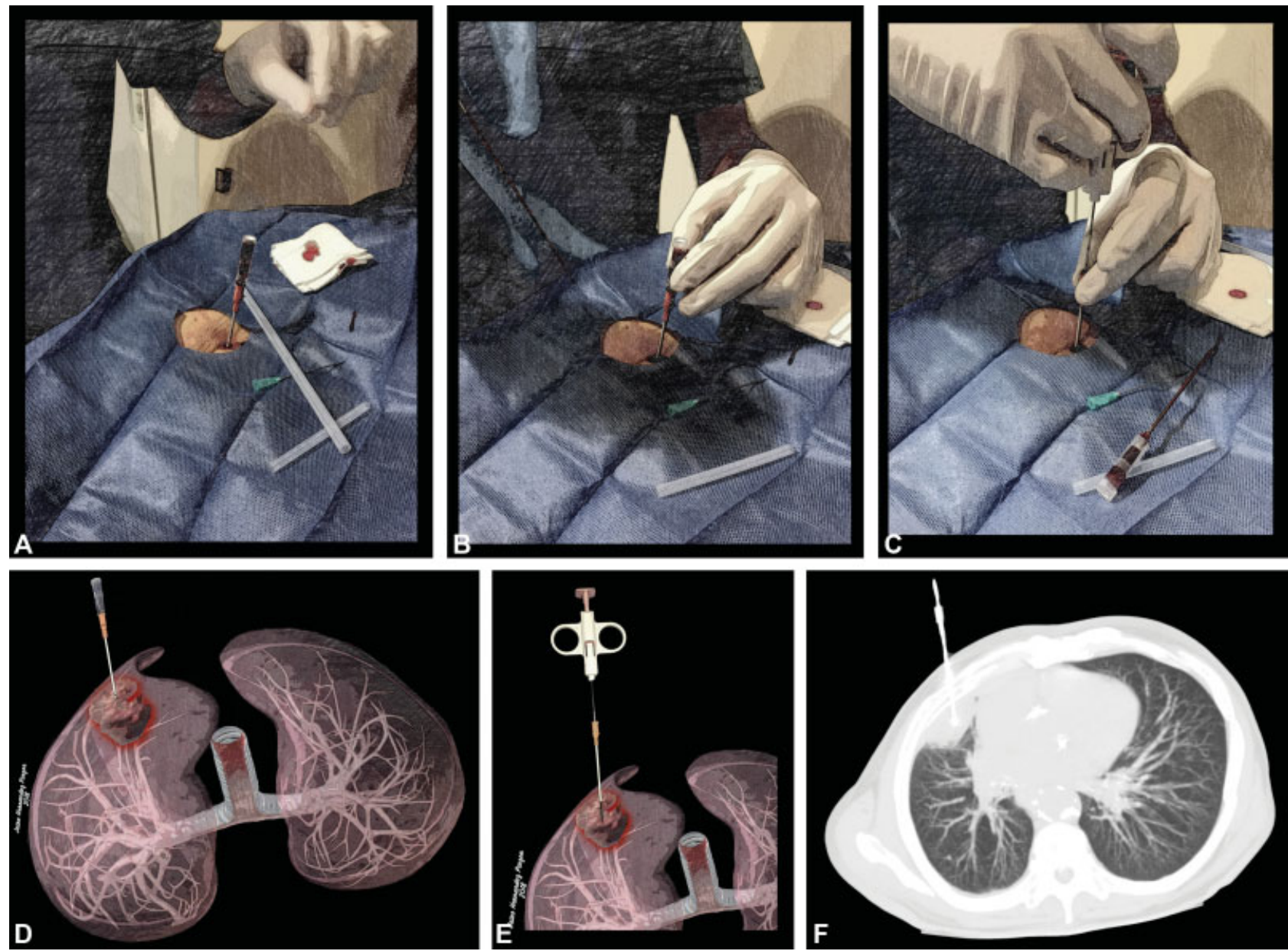

Fig. 1 Posterior a la aplicación de anestesia local y una pequeña incisión en la piel con bisturí, se insertó un Abocath 18G con su trayecto apuntando hacia la lesión pulmonar a nivel de la zona con menor espesor hasta llegar a la lesión (A), se realizó control tomográfico y se avanzó el Abocath hasta una distancia de $3 \mathrm{~mm}$ previo a la pleura parietal, posteriormente cuando existe seguridad sobre la trayectoria y la distancia que existe hasta la lesión, avanzamos el Abocath rápidamente perforando la pleura y llegando hasta la lesión (d), luego retiramos el estilete del Abocath (B) por donde introducimos la pistola de 18G (Cy E) y tomamos al menos dos muestras de tejido que son valoradas por el patólogo que se encontró presente durante el procedimiento. Finalmente realizamos un nuevo control tomográfico en busca de complicaciones (F). 

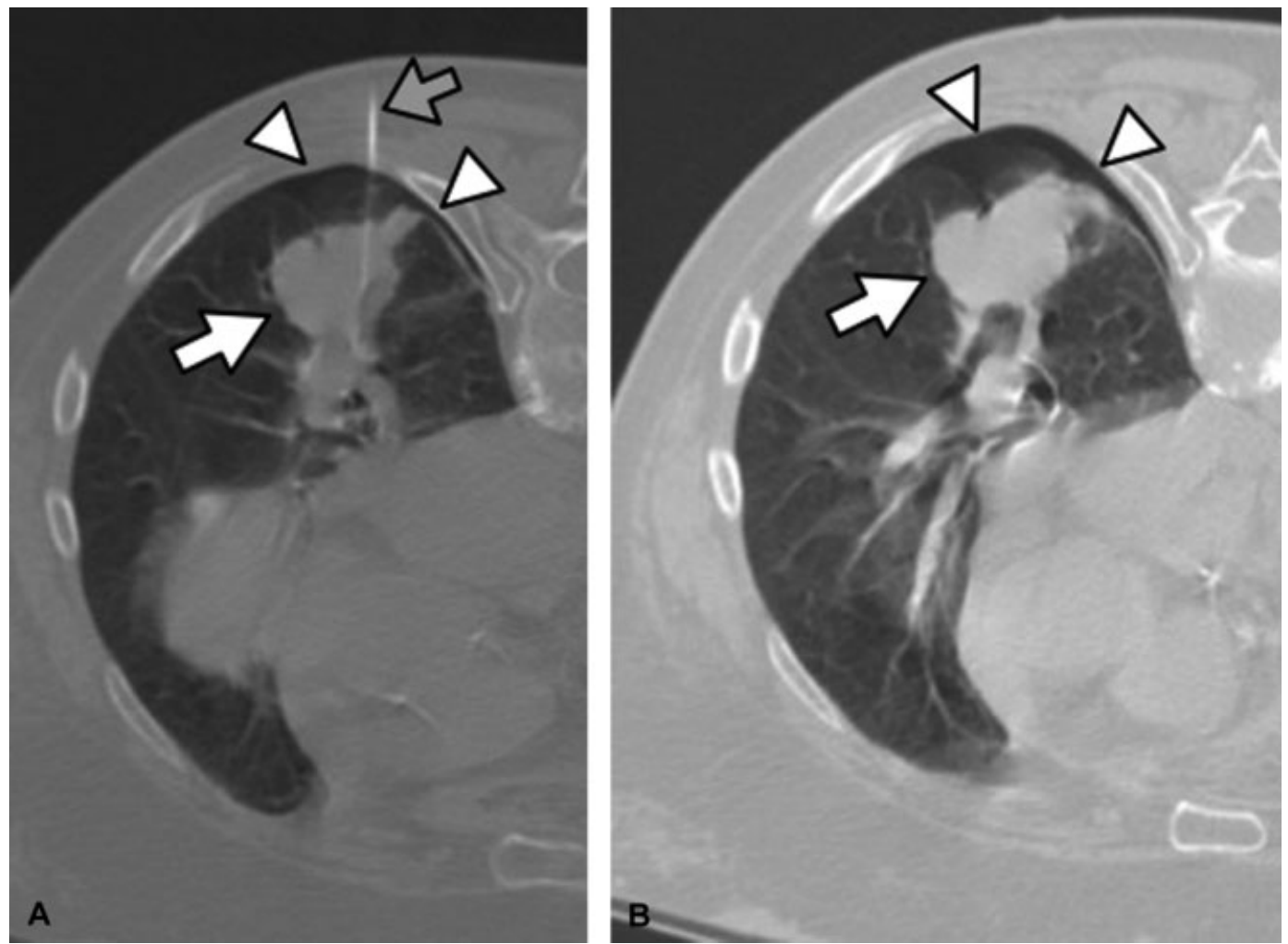

Fig. 2 Paciente con masa pulmonar (flecha blanca en A y B), sometido a PPT-TC, en plano axial. Se observó imagen laminar con densidad aérea en espacio pleural paralela a la pared torácica compatible con mínimo neumotórax (punta de flechas), nótese la aguja gruesa (flecha gris) atravesando la pared torácica y con extremo distal en la masa. TC plano axial (B) realizada 10 minutos después donde se observa incremento del contenido aéreo en el espacio pleural, hallazgo compatible con neumotórax leve.

control con TC, en ninguno de los casos se evidenció progresión del mismo.

\section{Resultados}

Se realizaron 26 punciones, la tasa de incidencia de complicaciones fue del 38,4\% en 1 año, el $26,9 \%$ presentó

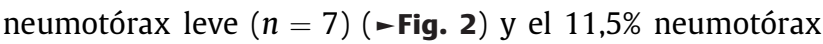
moderado $(n=3)$ (-Fig. 3 ), uno de los pacientes que presentó neumotorax leve desarrolló hemorragia alveolar difusa ( - Fig. 4), ese último caso requirió internación por 48 horas, tras las cuales presentó una adecuada evolución clínica. En ningún caso fue necesario tratamiento con tubo de avenamiento pleural.

La edad media de los pacientes fue de $66,5+/-14,0$ (rango 38-89 años), el $46,1 \%(n=12)$ eran tabaquistas al momento de la punción, la distancia de la lesión a la pared torácica interna varió desde $0 \mathrm{~mm}$ (lesiones subpleurales) a $31 \mathrm{~mm}$; el $19 \%(n=5)$ presentaban enfisema moderado y el $69 \%$ $(n=18)$ enfisema leve.

En cuanto al número de muestras obtenidas, en $61,5 \%$ $(n=16)$ se obtuvieron 3 muestras y en el $38,4 \%(n=10)$ se obtuvieron 2 muestras. Al analizar la presencia de complicaciones de acuerdo al número de muestras encontramos que el $29,9 \%(n=7)$ se encontraban en el grupo de 3 muestras y el $11,54 \%(n=3)$ se encontraban en el grupo de 2 muestras, diferencia que estadísticamente no fue significativa (test exacto de Fisher $=0,683$ ).
Los diagnósticos de anatomía patológica de cada lesión se detallan en la - Tabla 1. En el análisis estadístico univariado de los 26 pacientes, evidenciamos que los pacientes que presentaron complicaciones eran 14 años mayores (IC95\% 11,5 a 16,4 años) que los pacientes que no presentaron complicaciones $(61,1+/-3,6$ versus $75,1+/-2,4$ años $) \mathrm{t}$ (24) $=11,9403, p=0,0001$ (-Tabla 2).

\section{Discusión}

Los diferentes factores de riesgo para el desarrollo de complicaciones posteriores a la realización de una PPT-TC, es un tema que se ha estudiado con gran detalle sobre todo en la literatura anglosajona, sin embargo, en Argentina y Latinoamérica, existen pocos reportes sobre ese tema y menos aún series de casos punzados con aguja gruesa; teniendo en cuenta que actualmente existe una tendencia a punzar lesiones cada vez más pequeñas, ${ }^{1}$ hecho que probablemente incremente la tasa de complicaciones significativamente, creemos que nuestra experiencia en el tema a pesar de ser limitada, puede ser de utilidad para los especialistas en radiología que se inician en el campo del intervencionismo percutáneo.

El neumotórax ha sido la complicación reportada con mayor frecuencia posterior a la PPT-TC, Wagnetz y col. ${ }^{2}$ reportaron en 108 punciones 47 (44\%) casos de neumotórax leve y 14 (13\%) casos de neumotórax moderado con necesidad de drenaje pleural pos-procedimiento con tubo de avenamiento. 

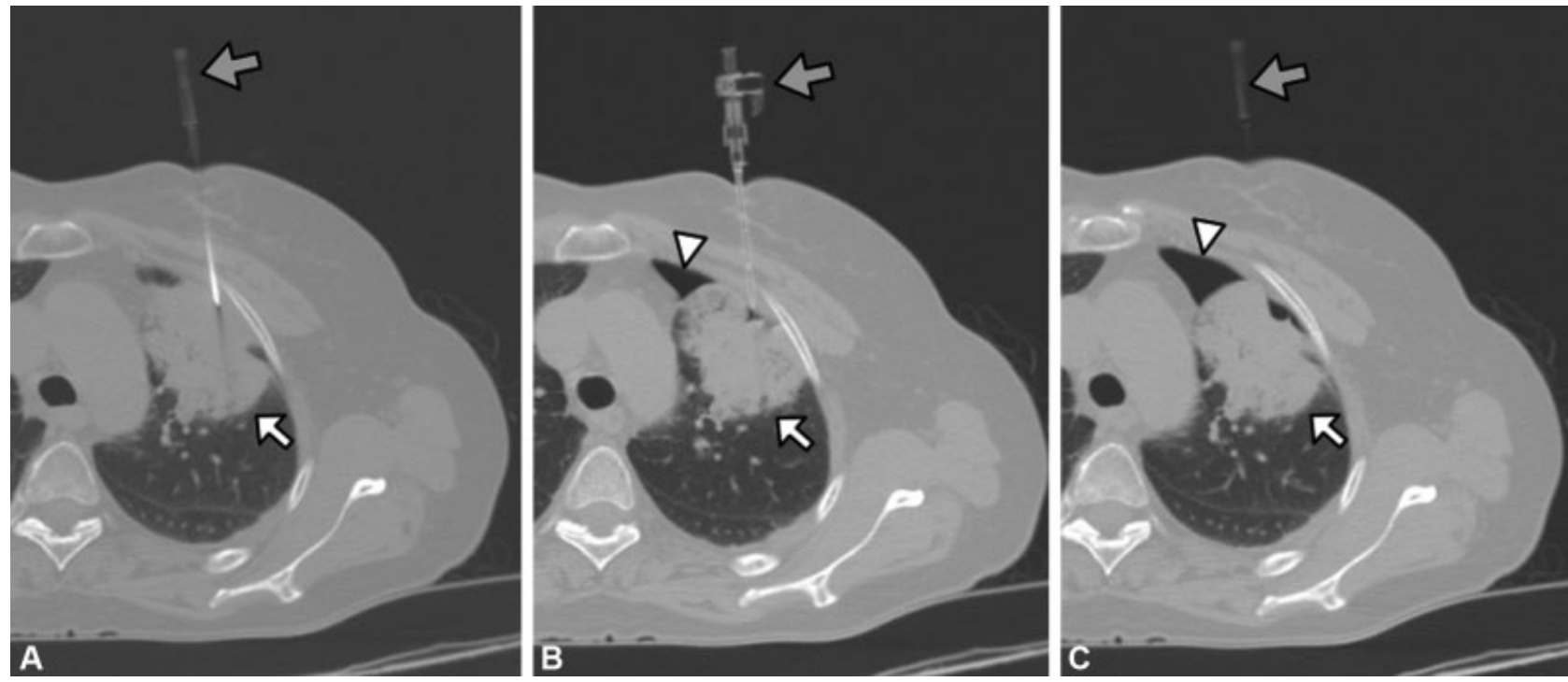

Fig. 3 Paciente con masa pulmonar en segmento anterior de lóbulo superior izquierdo (flecha blanca), en (A) TC plano axial, Abocath 18 (flecha gris). (B) TC plano axial realizada dos minutos posterior a la inserción del Abocath, se observó leve cantidad de contenido aéreo en el espacio pleural (punta de flecha blanca), dispositivo Trucut (flecha gris); (C) TC plano axial donde se evidencia aumento en la cantidad aérea contenida en la cavidad pleural, indicando neumotórax moderado.
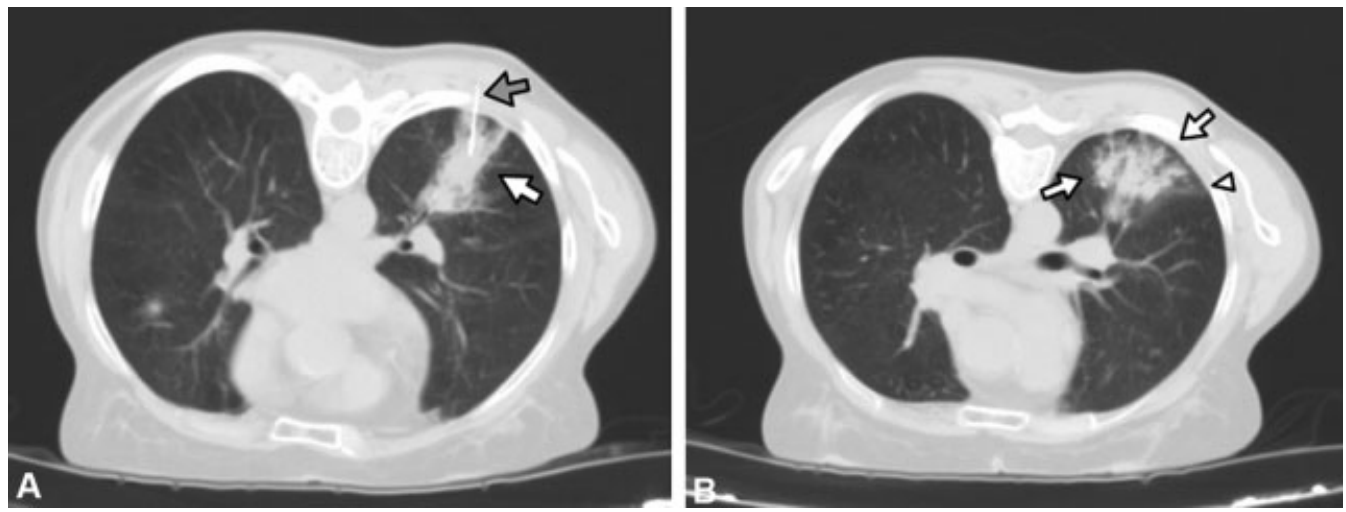

Fig. 4 Paciente en decúbito prono con lesión pulmonar en segmento apical de lóbulo inferior izquierdo (flecha blanca), (A) plano axial de TC se muestra Abocath 18 (flecha gris) con extremo distal en lesión pulmonar de bordes poco definidos los cuales presentan zonas de vidrio esmerilado. (B) TC de control posterior a finalizar el procedimiento, se observó aumento de las zonas con densidad en vidrio esmerilado (punta de flecha blanca) al igual que del componente denso de la lesión punzada (flechas blancas), esos hallazgos fueron interpretados como hemorragia alveolar difusa.

Tabla 1 Variables estudiadas de acuerdo a la presencia o ausencia de complicaciones; en la última columna se reportan los P-value de cada variable y la prueba estadística usada para su cálculo

\begin{tabular}{|l|l|l|l|}
\hline Variable & Con complicaciones & Sin complicaciones & P-Value \\
\hline Edad & 75,1 años $\pm 7,8$ (rango 64 a 89 años) & 61,1 años $\pm 14,57$ (rango 38 a 86 años) & t test $=0,0001$ \\
\hline Sexo & 6 mujeres y 4 hombres & 4 mujeres y 12 hombres & Chi2 $=0,074$ \\
\hline $\begin{array}{l}\text { Distancia a } \\
\text { la pleura }\end{array}$ & $4,75 \mathrm{~mm} \pm 8,5$ (rango 0 a $15 \mathrm{~mm}$ ) & $3,9 \mathrm{~mm} \pm 5,48$ (rango 0 a $31 \mathrm{~mm}$ ) & t test $=0,7816$ \\
\hline Enfisema & negativo (2), leve (7), moderado (1) & negativo (1) leve (11) moderado (4) & exacto de Fisher $=0,487$ \\
\hline Quick & $94,66 \pm 8,93$ (rango 77 a 100) & $95,61 \pm 7,27$ (rango 80 a 106) & t test $=0,808$ \\
\hline Kptt & 29,5 seg. $\pm 4,37$ (rango 25 a 36 seg.) & 31,61 seg. $\pm 4,51$ (rango 25 a 40 seg.) & t test $=0,3611$ \\
\hline Lóbulo & LSD (2), LM (1), LID (2), LSI (1), LII (4) & LSD (7), LM (1), LID (1), LSI (3), LII (4) & \\
\hline Total & 10 Pacientes & 16 Pacientes & \\
\hline
\end{tabular}


Tabla 2 Frecuencia de diagnósticos histopatológicos

\begin{tabular}{|l|l|l|}
\hline Diagnostico & Frecuencia & Porcentaje \\
\hline Adenocarcinoma & 6 & $26 \%$ \\
\hline Adenocarcinoma Lepídico & 2 & $8 \%$ \\
\hline Carcinoma Escamoso & 3 & $13 \%$ \\
\hline Carcinoma Células Pequeñas & 1 & $4 \%$ \\
\hline Fibrosis & 2 & $8 \%$ \\
\hline Tejido Graso & 1 & $4 \%$ \\
\hline Inflamación Crónica & 1 & $4 \%$ \\
\hline Linfoma células B & 1 & $4 \%$ \\
\hline $\begin{array}{l}\text { Metástasis Carcinoma } \\
\text { Células Claras }\end{array}$ & 1 & $4 \%$ \\
\hline Metástasis carcinoma Mama & 1 & $4 \%$ \\
\hline Metástasis carcinomatosa & 1 & $4 \%$ \\
\hline $\begin{array}{l}\text { Metástasis tumor Maligno } \\
\text { de Vaina Nerviosa Periférica }\end{array}$ & 1 & $4 \%$ \\
\hline Tejido Pulmonar Normal & 1 & $4 \%$ \\
\hline TBC & 1 & $4 \%$ \\
\hline
\end{tabular}

La segunda complicación más frecuente es la hemorragia, tanto intra como extrapulmonar; dentro de esos casos, puede existir volcado a la vía aérea y la aparición de signos como hemoptisis de diferentes grados de severidad; ${ }^{3}$ Ryan Tai, en un estudio reciente del 2015 , reportó una frecuencia de hemorragia del $41 \%$, con hemoptisis en $1,8 \%$ de los casos, en nuestra serie solo 1 paciente presentó esa complicación. ${ }^{1}$

Otra publicación sobre el tema, reportó en un periodo de 23 meses una tasa de complicaciones del 31\% (28 de 91 pacientes) y una tasa de hemoptisis de $2 \%$, con un porcentaje de mortalidad del $0,02 \%,{ }^{8}$ En nuestro estudio, no obtuvimos ningún desenlace fatal y nuestra incidencia acumulada de complicaciones fue del $38,46 \%$ en un año.

En nuestro medio vale la pena mencionar la experiencia de Ferreyra R. y col., ${ }^{9}$ quienes reportaron una serie de 155 casos atendidos en el hospital militar central de Buenos Aires, en donde utilizaron una aguja "Rotex II" en un periodo de 9 años aproximadamente, reportando 14 casos de neumotórax (9,03\%) de los cuales, 4 de ellos necesitaron inserción de tubo de avenamiento pleural.

Con respecto a Latinoamérica, en Chile, la doctora Besada $\mathrm{Cy}$ col. ${ }^{10}$ reportó una tasa de neumotórax del 13,7\% (21 de 153 pacientes), en 8 de los cuales se requirió la inserción de tubo de drenaje pleura; en esa serie de casos, se demostró una diferencia estadísticamente significativa en la distancia entre la pleura y la lesión pulmonar para los pacientes que presentaron complicaciones (con neumotórax $14,1 \pm 13,6 \mathrm{~mm}$ y sin neumotórax 7,5 $\pm 11,7 \mathrm{~mm}, \quad p=0,002)$ versus nuestros resultados (con neumotórax $4,75 \pm 8,5 \mathrm{~mm} \quad \mathrm{y}$ sin neumotórax 3,9 $\pm 5,48 \mathrm{~mm}, p=0,781)$, diferencia que se puede explicar por el bajo poder estadístico de nuestra serie debido al limitado número de pacientes que analizamos.

Se ha descrito que las principales variables relacionadas con el desarrollo de este tipo de complicación dependen, por un lado, de la técnica y del cuidado post-procedimiento y por el otro, de factores constitucionales del paciente y la lesión, siendo esos últimos el foco de nuestro trabajo. En nuestros pacientes, la única variable relacionada con el aumento en la incidencia de complicaciones fue la edad, resultado que concuerda con lo reportado en estudios previos, ${ }^{11,12}$ aunque en los estudios citados se encontraron otras variables relacionadas. Respecto a ese punto, vale la pena mencionar la serie de pacientes reportada por Snag Min lee y col. en el 2014, mostrando una incidencia de neumotórax del $17 \%$, según su experiencia, las variables que aumentaron el riesgo de neumotórax significativamente además de la edad fueron: localización (lóbulos inferiores), sexo (masculino), número de pasajes del dispositivo trucut (mayor a dos), y el enfisema en el trayecto de punción.

Es muy probable que nuestras diferencias respecto a los estudios mencionados se deban al bajo número de pacientes analizados, por lo que tenemos que hacer la salvedad que esta comunicación no busca controvertir los resultados reportados por estudios con un poder estadístico mayor, sino más bien, ser un primer paso para la elaboración de investigaciones prospectivas con diseños metodológicos más robustos; en principio, planteamos la realización de un análisis multivariado, sin embargo, para este fin, hubiésemos necesitado 10 eventos por cada variable predictiva (aproximadamente 60 pacientes con complicaciones para analizar las variables estudiadas), y aunque desde el año 2006 se sabe que esa regla es en extremo conservadora, ${ }^{13}$ decidimos no incluir esa información en el presente manuscrito a fin de no generar confusión en los lectores.

Otra de las limitaciones encontradas se debe a que nuestra institución es un centro de referencia nacional en cuanto a patología oncológica pulmonar, existiendo la posibilidad de que la muestra de pacientes con la que realizamos los análisis estadísticos no sea representativa de la población general a la que se le realiza PPT-TC, ya que cuando un centro médico posee un equipo de profesionales reconocidos en determinada área, tiende a atraer casos complejos o poco habituales.

\section{Conclusión}

La PPT-TC es un procedimiento que aunque pueda parecer simple, presenta una tasa considerable de complicaciones no severas, por lo cual los médicos especialistas en diagnóstico por imágenes que lo realizan deben considerar que los resultados finales de morbilidad pueden estar influenciados significativamente por variables inherentes al paciente, como lo fue la edad en nuestra serie de casos.

\section{Responsabilidades Éticas}

Protección de personas y animales. Los autores declaran que para esta investigación no se han realizado experimentos en seres humanos ni en animales.

Confidencialidad de los datos. Los autores declaran que han seguido los protocolos de su centro de trabajo sobre la publicación de datos de pacientes. 
Derecho a la privacidad y consentimiento informado. Los autores declaran que en este artículo no aparecen datos de pacientes.

\section{Conflicto de Intereses}

Los autores declaran no tener ningún conflicto de interés, con excepción del Dr. Kozima que declara como posible conflicto de interés formar parte de la Comisión Directiva de la SAR y el Dr. Hernández Pinzón, que declara como posible conflicto de interés integrar el Comité de Redacción de la RAR.

\section{Bibliografía}

1 Tai R, Dunne RM, Trotman-Dickenson B, et al. Frequency and severity of pulmonary hemorrhage in patients undergoing percutaneous CTguided transthoracic lung biopsy: single-institution experience of 1175 cases. Radiology 2016;279(01):287-296

2 Wagnetz U, Menezes RJ, Boerner S, et al. CT screening for lung cancer: implication of lung biopsy recommendations. AJR Am J Roentgenol 2012;198(02):351-358

3 Schneider F, Smith MA, Lane MC, Pantanowitz L, Dacic S, Ohori NP. Adequacy of core needle biopsy specimens and fine-needle aspirates for molecular testing of lung adenocarcinomas. Am J Clin Pathol 2015;143(02):193-200, quiz 306

4 Lindeman NI, Cagle PT, Beasley MB, et al. Molecular testing guideline for selection of lung cancer patients for EGFR and ALK tyrosine kinase inhibitors: guideline from the College of American Pathologists, International Association for the Study of Lung
Cancer, and Association for Molecular Pathology. J Thorac Oncol 2013;8(07):823-859

5 Anzidei M, Porfiri A, Andrani F, et al. Imaging-guided chest biopsies: techniques and clinical results. Insights Imaging 2017; 8(04):419-428

6 Winokur RS, Pua BB, Sullivan BW, Madoff DC. Percutaneous lung biopsy: technique, efficacy, and complications. Semin Intervent Radiol 2013;30(02):121-127

7 Arcila ME, Oxnard GR, Nafa K, et al. Rebiopsy of lung cancer patients with acquired resistance to EGFR inhibitors and enhanced detection of the T790M mutation using a locked nucleic acid-based assay. Clin Cancer Res 2011;17(05): $1169-1180$

8 Ferretti GR, Busser B, de Fraipont F, et al. Adequacy of CT-guided biopsies with histomolecular subtyping of pulmonary adenocarcinomas: influence of ATS/ERS/IASLC guidelines. Lung Cancer 2013;82(01):69-75

9 Ferreyra R, Lizzi L. Punción transparietal de pulmón con informe citopatológico inmediato. Rev Argent Med Respir 2005;1:41-47

10 Besa C, Huele A, Bächler P, Cruz F. Utilidad de la biopsia percutánea core guiada por tomografía computada (TC) en lesiones pulmonares: experiencia de 7 años. Rev Med Chil 2013;141 (04):449-456

11 Collings CL, Westcott JL, Banson NL, Lange RC. Pneumothorax and dependent versus nondependent patient position after needle biopsy of the lung. Radiology 1999;210(01):59-64

12 Heck SL, Blom P, Berstad A. Accuracy and complications in computed tomography fluoroscopy-guided needle biopsies of lung masses. Eur Radiol 2006;16(06):1387-1392

13 Vittinghoff E, McCulloch CE. Relaxing the rule of ten events per variable in logistic and Cox regression. Am J Epidemiol 2007;165 (06):710-718 